\title{
FACTOR IDEALS \\ OF SOME REPRESENTATION ALGEBRAS
}

\author{
W. D. WALLIS
}

(Received 19 December 1966)

Throughout this paper $\mathscr{F}$ is an algebraically closed field of characteristic $p(\neq 0)$ and $\mathscr{G}$ is a finite group whose order is divisible by $p$. We define in the usual way an $\mathscr{F}$-representation of $\mathscr{G}$ (or $\mathscr{F} \mathscr{G}$-representation) and its corresponding module. The isomorphism class of the $\mathscr{F} \mathscr{G}$-representation module $\mathscr{M}$ is written $\{\mathscr{K}\}$ or, where no confusion arises, $M . A(\mathscr{G})$ denotes the $\mathscr{F}$-representation algebra of $\mathscr{G}$ over the complex field $\mathscr{C}$ (as defined on pages 73 and 82 of [6]).

J. A. Green [6] and S. B. Conlon [5] have shown that $A(\mathscr{G})$ is semisimple if and only if the algebras $W_{\mathscr{g}}(\mathscr{G})$ (see Section 1) are all semisimple, where $\mathscr{D}$ runs over the set of distinct (to within $\mathscr{G}$-conjugacy) $p$-subgroups of $\mathscr{G}$.

The semisimplicity of $W_{\mathscr{g}}(\mathscr{G})$ is known to hold when $\mathscr{D}$ is cyclic and in the special case where $p=2$ and $\mathscr{D}$ is a sylow subgroup of $\mathscr{G}$ and is isomorphic to the elementary abelian group on two generators $\mathscr{V}_{4}$. I have considered the case where $\mathscr{D}$ is this group but the sylow condition does not hold; it is shown that $W_{\mathscr{g}}(\mathscr{G})$ is semisimple when $\mathscr{G}$ is the alternating group on six or seven symbols $\left(\mathscr{A}_{6}\right.$ or $\left.\mathscr{A}_{7}\right)$. It is also shown that $A\left(\mathscr{A}_{6}\right)$ is semisimple if and only if $A\left(\mathscr{A}_{7}\right)$ is. Use is made of Conlon's results. [3] on $A\left(\mathscr{V}_{4}\right)$ and $A\left(\mathscr{A}_{4}\right)$.

Section 1 of the paper is introductory. In the second section certain properties of alternating groups are indicated; the semisimplicity of $A\left(\mathscr{A}_{5}\right)$ is proven and those groups $\mathscr{P}$ for which $W_{\mathscr{B}}\left(\mathscr{A}_{6}\right)$ is isomorphic to $W_{\mathscr{G}}\left(\mathscr{A}_{7}\right)$ are listed. The remainder of the paper is concerned with the semisimplicity of $W_{\boldsymbol{y}_{k}}\left(\mathscr{A}_{k}\right)$ for $k=6$ or 7 . Certain results derived in Section 3 concerning the decomposition of induced modules are applied in the next section to the $\mathscr{V}_{4}$-projective $\mathscr{N}_{6}$ and $\mathscr{N}_{7}$-modules $\left(\mathscr{N}_{k}\right.$ is the $\mathscr{A}_{k}$-normalizer of $\left.\mathscr{V}_{4}\right)$; the generators of $A_{\mathscr{r}_{t}}\left(\mathscr{N}_{k}\right)$ modulo the projective ideal are calculated. In the final sections the structures of $W_{\boldsymbol{r}_{4}}\left(\mathscr{N}_{6}\right)$ and $W_{\boldsymbol{r}_{4}}\left(\mathscr{N}_{7}\right)$ are found; semisimplicity follows.

This research was carried out at the University of Sydney. I wish to express my thanks to Dr. S. B. Conlon for his help with this work. 


\section{Properties of representation algebras}

We shall say an algebra $\mathscr{A}$ over the complex field $\mathscr{C}$ is semisimple ${ }^{1}$ if, for every non-zero element $A$ of $\mathscr{A}$, there is an algebra homomorphism $\phi_{A}: \mathscr{A} \rightarrow \mathscr{C}$, with $A \phi_{A} \neq 0 .{ }^{2}$ If the radical of $\mathscr{A}$ is defined as the intersection $\cap \mathscr{M}$ of the maximal ideals $\mathscr{M}$ of $\mathscr{A}$, such that $\mathscr{A} / \mathscr{M} \cong \mathscr{C}$, then a necessary and sufficient condition for the semisimplicity of $\mathscr{A}$ is that $\mathscr{A}$ should have zero radical. ${ }^{3}$

If $A(\mathscr{G})$ is as defined above, and $\mathscr{D} \leqq \mathscr{G}$, we define $A_{\mathscr{D}}(\mathscr{G})$ and $W_{\mathscr{D}}(\mathscr{G})$ as in [6]. We write $W_{\mathscr{G}}(\mathscr{G})=A_{\mathscr{S}}(\mathscr{G})$ where $\mathscr{I}$ is the group with one element. It should be noted that $W_{\mathscr{D}}(\mathscr{G})$ is trivial unless $\mathscr{D}$ is a $p$-group.

If $\mathscr{H}$ is a subgroup of $\mathscr{G}$, then the following results hold:

(1) Theorem [5]. $A_{\mathscr{*}}(\mathscr{G}) \cong \oplus W_{\mathscr{g}}(\mathscr{G})$, where $\oplus$ is the algebra direct sum over all the non-G-conjugate $p$-subgroups $\mathscr{D}$ of $\mathscr{G}$ with $\mathscr{D} \leqq g \mathscr{H}$.

(2) Corollary. $A_{*}(\mathscr{G})$ is semisimple if and only if each $W_{\mathscr{g}}(\mathscr{G})$ is.

It follows from (1) and (2) that to discuss semisimplicity we need only discuss the semisimplicity of the algebras $W$. We make use of the following result.

(3) THEOREM $[6$, p. 81]. If $\mathscr{D} \leqq \mathscr{V} \leqq \mathscr{H} \leqq \mathscr{G}$, where $\mathscr{N}$ is the $\mathscr{G}-$ normalizer of $\mathscr{D}$, then

$$
W_{\mathscr{D}}(\mathscr{H}) \cong W_{\mathscr{D}}(\mathscr{G}) \text {. }
$$

In other words we need only consider the normalizer of $\mathscr{D}$ in $\mathscr{G}$, rather than $\mathscr{G}$.

$W_{\mathcal{G}}(\mathscr{G})$ is always semisimple; in fact as $\mathscr{F}$ is algebraically closed it is the direct sum of $r$ copies of $\mathscr{C}$ where $r$ is the number of $p$-regular conjugacy classes in $\mathscr{G}$ [3, p. 85].

Finally we note that there is no loss of generality in considering $\mathscr{F}$ algebraically closed. If it were not, then let $\mathscr{F} *$ be the closure of $\mathscr{F}$, and write $A^{*}(\mathscr{G}), W_{\mathscr{D}}^{*}(\mathscr{G})$ for the algebras derived in this case. Then from p. 80 of [4] we see that the semisimplicity of $A^{*}$ (or $W^{*}$ ) ensures that of $A$ (or $W$ ).

\section{Alternating groups}

If we take $\mathscr{A}_{r}$ as a permutation group on the first $r$ positive integers in the usual way we can write $\mathscr{A}_{r-1}<\mathscr{A}_{r}$, where $\mathscr{A}_{r-1}$ is obtained by omitting all elements of $\mathscr{A}_{\text {r }}$ which properly permute one specific integer.

1 Also called $G$-semisimple [3, p. 84].

2 All mappings except permutations and representations are written on the right.

3 See p. 84 of [3]. 
Suppose $\mathscr{D} \leqq \mathscr{A}_{r-1}$ and suppose the normalizer $\mathscr{N}\left(\mathscr{D} ; \mathscr{A}_{r}\right)$ of $\mathscr{D}$ in $\mathscr{A}_{r}$ satisfies $\mathscr{N}\left(\mathscr{D} ; \mathscr{A}_{r}\right) \leqq \mathscr{A}_{r-1}$. Then, by (3),

$$
W_{\mathscr{Q}}\left(\mathscr{A}_{r}\right) \cong W_{\mathscr{D}}\left(\mathscr{A}_{r-1}\right) \text {. }
$$

This sort of result interests us because it provides a link between $A\left(\mathscr{A}_{r}\right)$ and $A\left(\mathscr{A}_{r-1}\right)$, so we will investigate when these conditions hold. To do this we introduce the idea of a permutation acting on an integer.

We say the permutation $P$ acts on the integer $i$ if, when $P$ is considered as a mapping of the integers, $P(i) \neq i$, and a subgroup $\mathscr{P}<\mathscr{A}_{r}$ acts on $i$ if there is a member of $\mathscr{P}$ which acts on $i$. Thus the positive integers $\leqq r$ are divided into two disjoint classes: $\alpha_{r}(\mathscr{P})$, the set of integers acted on by $\mathscr{P}$, and $\beta_{r}(\mathscr{P})$, the set of integers invariant under $\mathscr{P}$. Then it is easy to see that $\mathscr{N}\left(\mathscr{P} ; \mathscr{A}_{r}\right)$ consists those even permutations $N$ of the form $N=N_{1} N_{2}$, where $N_{1} \in \mathscr{N}\left(\mathscr{P} ; \mathscr{S}_{a}\right)$ and $N_{2} \in \mathscr{S}_{r-a}, a$ being the order of $\alpha_{r}(\mathscr{P}), \mathscr{S}_{a}$ the symmetric group on the elements of $\alpha_{r}(\mathscr{P})$, and $\mathscr{S}_{r-a}$ the symmetric group on the elements of $\beta_{r}(\mathscr{P})$.

If $a=r-1$ then $\mathscr{S}_{r-a}$ consists the identity element only, and in this case $\mathscr{N}\left(\mathscr{P} ; \mathscr{A}_{r}\right)=\mathscr{N}\left(\mathscr{P} ; \mathscr{A}_{r-1}\right) \leqq \mathscr{A}_{r-1}$. This is the condition we wanted.

(4) TheOREM. If $\mathscr{P}$ is a subgroup of $\mathscr{A}_{p n+1}$ which acts on $p n$ symbols then

$$
\mathscr{N}\left(\mathscr{P} ; \mathscr{A}_{p n+1}\right)=\mathscr{N}\left(\mathscr{P} ; \mathscr{A}_{p n}\right)
$$

and so

$$
W_{g}\left(\mathscr{A}_{p n+1}\right) \cong W_{\mathscr{P}}\left(\mathscr{A}_{p n}\right) \text {. }
$$

$\left(\mathscr{A}_{p n}\right.$ is the alternating group on the symbols acted on by $\left.\mathscr{P}\right)$.

We have considered $\mathscr{P}$ acting on $n p$ symbols because of the following result:

(5) Lemma. If $\mathscr{P}$ is a permutation group of order $p^{m}$, where $p$ is prime, then $\mathscr{P}$ acts on exactly pn symbols for some integer $n$.

Proof. Consider the orbit $\mathscr{P}(i)$ of the symbol $i$ under $\mathscr{P}$.

$$
\mathscr{P}(i)=\{j: P(i)=j \text { for some } P \in \mathscr{P}\} .
$$

Then, by theorem 3.2 on p. 5 of [9], the order $|\mathscr{P}(i)|$ of $\mathscr{P}(i)$ divides that of $\mathscr{P}$. From the fact that $\mathscr{P}$ is a $p$-group, $|\mathscr{P}(i)|$ must be a power of $p$.

The set of symbols acted upon by $\mathscr{P}$ is, by definition,

that is

$$
\{i:|\mathscr{P}(i)| \neq 1\} \text {, }
$$

$$
\cup \mathscr{P}(j),
$$

the union being taken over all $j$ such that $|\mathscr{P}(j)| \neq 1$. Since any two distinct orbits are disjoint, this set has order 


\section{$\sum|\mathscr{P}(j)|$,}

and every term in the sum is a power of $p$, not $p^{0}$. Thus $p$ divides the sum, giving the result, except for the trivial case where $\mathscr{P}$ acts on no symbols, in which case $m=0$ and we can put $n=0$.

In particular a sylow $p$ - subgroup of $\mathscr{A}_{r}$ must act on $n p$ symbols where $n$ is the largest integer such that $n p \leqq r$, so a sylow $p$-subgroup of $\mathscr{A}_{n n+1}$ acts on exactly $p n$ symbols and satisfies the conditions of (4).

We shall consider the case $p=2$. With $n=2$ we can apply (4) to $\mathscr{A}_{4}$ and $\mathscr{A}_{5}$. Every 2-subgroup of $\mathscr{A}_{5}$ except $\mathscr{I}$ acts on $4(=p n)$ symbols, so if $\mathscr{P}$ is such a group,

$$
W\left(\mathscr{A}_{5}\right) \cong W_{\mathscr{S}}\left(\mathscr{A}_{4}\right) \text {. }
$$

$A\left(\mathscr{A}_{4}\right)$ is semisimple [3, p. 97], so each $W_{g}\left(\mathscr{A}_{4}\right)$ is semisimple by (2), and so each $W_{\mathscr{O}}\left(\mathscr{A}_{5}\right)$ is semisimple. Moreover $W_{\mathscr{f}}\left(\mathscr{A}_{5}\right)$ is semisimple. As any 2-subgroup of $\mathscr{A}_{5}$ is of the same type as $\mathscr{P}$ or is $\mathscr{I}$, we see by (2) that $A\left(\mathscr{A}_{5}\right)$ is semisimple.

If $n=3$ we have $\mathscr{A}_{8}$ and $\mathscr{A}_{7}$. If we write

$$
\begin{array}{lll}
U=(13)(24) & X=(123) & Z=(1234)(56) \\
V=(14)(23) & Y=(567) & T=(12)(56)
\end{array}
$$

then the distinct 2-subgroups of $\mathscr{A}_{7}$ are $\mathscr{I}, \mathscr{Z}_{2}, \mathscr{V}, \mathscr{V}^{\prime}, \mathscr{Z}_{4}$ and $\mathscr{P}$, and their $\mathscr{A}_{7}$-conjugates, where

$$
\begin{aligned}
\mathscr{Z}_{2} & =\langle U\rangle \\
\mathscr{V} & =\langle U, V\rangle \\
\mathscr{V}^{\prime} & =\langle U V, T\rangle \\
\mathscr{Z}_{4} & =\langle Z\rangle \\
\mathscr{P} & =\langle Z, V\rangle .
\end{aligned}
$$

These are also the 2-subgroups of $\mathscr{A}_{6}$.

We shall prove that $W_{\mathscr{r}}\left(\mathscr{A}_{6}\right)$ and $W_{\mathscr{r}}\left(\mathscr{A}_{7}\right)$ are semisimple. That $W_{\mathscr{g}}(\mathscr{G})$ is semisimple when $\mathscr{D}=\mathscr{I}$ has already been noted; that it is so when $\mathscr{D}$ is cyclic has been shown in [8]. From (4) we see that

$$
W_{\mathscr{V}^{\prime}}\left(\mathscr{A}_{6}\right) \cong W_{\mathscr{V}^{\prime}}\left(\mathscr{A}_{7}\right) \text { and } W_{\mathscr{g}}\left(\mathscr{A}_{6}\right) \cong W_{\mathscr{F}}\left(\mathscr{A}_{7}\right) \text {, }
$$

since both these subgroups act on $\mathbf{6}(=n p)$ symbols. Collecting these results and applying (2) we see that $A\left(\mathscr{A}_{7}\right)$ is semisimple if and only if $A\left(\mathscr{A}_{6}\right)$ is.

Even more can be said. There is an isomorphism between $\mathscr{N}\left(\mathscr{V} ; \mathscr{A}_{6}\right)$ and $\mathscr{N}\left(\mathscr{V}^{\prime} ; \mathscr{A}_{6}\right)$ which carries $\mathscr{V}$ onto $\mathscr{V}^{\prime}$, and so $W_{\mathscr{V}^{\prime}}\left(\mathscr{A}_{6}\right) \cong W_{\mathscr{V}}\left(\mathscr{A}_{6}\right)$ and is semisimple. Therefore $A\left(\mathscr{A}_{6}\right)$ and $A\left(\mathscr{A}_{7}\right)$ are semisimple if and only if $W_{\mathscr{G}}\left(\mathscr{A}_{6}\right)$ is. As $\mathscr{N}\left(\mathscr{P}_{\mathscr{A}_{6}}\right)$ is $\mathscr{P}$, we can say $A\left(\mathscr{A}_{6}\right)$ and $A\left(\mathscr{A}_{7}\right)$ are semisimple if and only if $W_{\mathscr{g}}(\mathscr{P})$ is semisimple. 
It remains to show that $W_{\mathscr{r}}\left(\mathscr{A}_{6}\right)$ and $W_{\mathscr{r}}\left(\mathscr{A}_{7}\right)$ are semisimple. By (3) we need only consider $W_{\mathscr{V}}\left(\mathscr{N}_{\boldsymbol{B}}\right)$ and $W_{\mathscr{V}}\left(\mathscr{N}_{7}\right)$ where $\mathscr{N}_{r}$ is $\mathscr{N}\left(\mathscr{V} ; \mathscr{A}_{r}\right)$ and

$$
\begin{aligned}
& \mathscr{N}_{6}=\langle U, V, X, T\rangle, \\
& \mathscr{N}_{7}=\langle U, V, X, T, Y\rangle .
\end{aligned}
$$

\section{Decomposition of induced modules}

Suppose $\mathscr{H}$ is a normal subgroup of $\mathscr{G}$, and let $\mathscr{L}$ be an indecomposable $\mathscr{F} \mathscr{H}$-representation module with $\mathscr{G}$-stabilizer $\mathscr{S}$. Then we know [2, p. 162] that $\mathscr{L}^{\mathscr{S}}$ decomposes as does a certain twisted group algebra $\mathscr{I}(\mathscr{S} \mid \mathscr{H})$ on $\mathscr{S} \mid \mathscr{H}$ over $\mathscr{F}$. To be specific, suppose $\mathscr{L}^{\mathscr{S}} \cong \mathscr{L}_{1} \oplus \mathscr{L}_{2} \oplus \cdots \oplus \mathscr{L}_{m}$ and $\mathscr{I}(\mathscr{S} \mid \mathscr{H}) \cong \mathscr{I}_{1} \oplus \mathscr{I}_{2} \oplus \cdots \oplus \mathscr{I}_{n}$, where the $\mathscr{L}_{i}$ are indecomposable submodules and the $\mathscr{I}_{i}$ are indecomposable left ideals. Then we can reorder the $\mathscr{I}_{i}$ so that

$$
\begin{aligned}
m & =n \\
\mathscr{L}_{i} & \cong \mathscr{L}_{j} \text { if and only if } \mathscr{I}_{i} \cong \mathscr{I}_{j} \\
\operatorname{dim}_{\mathscr{F}} \mathscr{L}_{i} & =\operatorname{dim}_{\mathscr{F}} \mathscr{I}_{i} \cdot \operatorname{dim}_{\mathscr{F}} \mathscr{L} .
\end{aligned}
$$

Furthermore $\mathscr{L}_{i}^{*}$ is indecomposable.

(7) ThEOREM. If $\mathscr{S}=\mathscr{H} \times \mathscr{W}$, then

$$
\mathscr{L}^{\mathscr{S}} \cong \stackrel{\oplus}{1}_{1}^{n} \mathscr{W}_{i} \otimes_{F x^{\mathscr{L}}}
$$

is a decomposition into indecomposables, where

$$
\mathscr{F} \mathscr{W}=\stackrel{n}{\oplus}_{1}^{\oplus} \mathscr{W}_{i}
$$

is a decomposition into indecomposable left ideals. $\left(\mathscr{W}_{i} \otimes{ }_{\mathfrak{F}} \mathscr{L}\right.$ is defined as the subset of $\mathscr{F} \mathscr{S} \otimes \mathscr{W}^{\mathscr{L}}$ consisting of the elements $W \otimes L$, where $W \in \mathscr{W}_{i}$ and $L \in \mathscr{L}$.)

Proof. It is clear that the sum is direct and equals $\mathscr{L}^{\mathscr{S}}$ and that the summands are $\mathscr{F} \mathscr{S}$-representation modules. $\mathscr{I}(\mathscr{S} \mid \mathscr{H})$ is $\mathscr{F}(\mathscr{S} \mid \mathscr{H})$ in this case, so by (6) $\mathscr{L}^{\mathscr{S}}$ must split into exactly $h$ indecomposable parts. By the Krull-Schmidt theorem these must be the $\mathscr{W}_{i} \otimes \mathscr{L}$.

Write $\mathscr{X}$ for the alternating group on $\{5,6, \cdots, k\}$. Then (7) applies when $\mathscr{G}=\mathscr{V} \times \mathscr{X}$ or $\mathscr{A}_{4} \times \mathscr{X}$, which are the cases we will need.

\section{4. $\mathscr{V}$-projective representations of $\mathscr{N}_{\boldsymbol{k}}$}

The indecomposable $\mathscr{V}$-projective $\mathscr{F}_{\mathscr{N}_{k}}$-representation modules are just the indecomposable parts of the modules $\mathscr{L}^{\boldsymbol{r}_{k}}$ where $\mathscr{L}$ is an $\mathscr{F} \mathscr{V}$ representation module. 
The indecomposable $\mathscr{F} \mathscr{V}$-representation module isomorphism classes are known ([1], [7]). In the notation of Conlon [3] the classes are $A_{0}$, $A_{n}, B_{n}, C_{n}(f)$ and $D$, where $n$ ranges through the positive integers and $f$ through $\mathscr{F} \cup\{\infty\}$. Typical representations are given on pp. 86-7 of [3]; we shall denote this representative of $A_{n}$ by $\mathscr{A}_{n}$, and similarly for the others. The stabilizers are given in (8):

\begin{tabular}{lll}
\hline Module & \multicolumn{2}{c}{ Generators of Stabilizer } \\
\hline $\mathscr{A}_{n}, \mathscr{B}_{n}, \mathscr{D}$ & in $\mathscr{N}_{0}$ & in $\mathscr{N}_{7}$ \\
$\mathscr{C}_{n}(0)$ & $U, V, X, T$ & $U, V, X, T, Y$ \\
$\mathscr{C}_{n}(1)$ & $U V, T X$ & $U, V, T X, Y$ \\
$\mathscr{C}_{n}(\infty)$ & $U, V, T$ & $U, V, T, Y$ \\
$\mathscr{C}_{n}(\omega), \mathscr{C}_{n}\left(\omega^{2}\right)$ & $U, V, T X^{2}$ & $U, V, T X^{2}, Y$ \\
$\mathscr{C}_{n}(\mathrm{f})$ & $U, V, X$ & $U, V, X, Y$ \\
\hline
\end{tabular}

where $\omega$ is a primitive cube root of unity in $\mathscr{F}$ and $f$ ranges over all values not already listed. The stabilizer in $\mathscr{N}_{k}$ may be derived from that in $\mathscr{N}_{6}$ as noted in Section 3.

The representations of $\mathscr{A}_{4}$ are also known [3]; their classes are $A_{0}^{a}$, $\bar{A}_{n}^{a}, \bar{B}_{n}^{a}, \bar{C}_{n}^{a}(g), C_{n}^{*}(f), D^{a}$, where $n$ ranges through the positive integers, $a$ through the integers modulo $3, g$ through $\left\{\omega, \omega^{2}\right\}$ and $f$ through a set of representatives of the equivalence classes of $\mathscr{F}$ under the relation

$$
f \sim 1+\frac{1}{f} \sim \frac{1}{1+f}, \quad \text { except } \omega \text { and } \omega^{2} .
$$

If we write $\overline{\mathscr{A}}_{n}^{a}$ for a representative module of the class $\bar{A}_{n}^{a}$, and so on, then $\overline{\mathscr{L}}^{a}$ may be taken as an extension of $\mathscr{L} ; \overline{\mathscr{C}}_{n}^{*}(f)$ may be taken as $\left(\mathscr{C}_{n}(f)\right)^{\mathscr{\alpha}_{4}}$. We follow Conlon's convention that $\overline{\mathscr{A}}_{0}^{a}$ yields the representation

and

$$
U \rightarrow 1, V \rightarrow 1, X \rightarrow \omega^{a}
$$

$$
\overline{\mathscr{A}}_{0}^{a} \otimes \overline{\mathscr{L}}^{b} \cong \overline{\mathscr{L}}^{a+b} \text {. }
$$

Then if $\overline{\mathscr{L}}^{0}$ gives the representation $X \rightarrow \lambda(X), \overline{\mathscr{L}}^{a}$ gives $X \rightarrow \omega^{a} \lambda(X)$. Suitable matrices $\lambda(X)$ are known [3] for all cases except $\mathscr{C}_{n}(\omega)$ and $\mathscr{C}_{n}\left(\omega^{2}\right)$; and the author has found that a suitable matrix to extend $\mathscr{C}_{n}(\omega)$ is $M_{1} \oplus M_{2}$, where

$$
\begin{aligned}
& M_{1} \text { has }(i, j) \text { element } \omega^{i+j+1}\left(\begin{array}{l}
i-2 \\
j-2
\end{array}\right) \\
& M_{2} \text { has }(i, j) \text { element } \omega^{i+j+2}\left(\begin{array}{l}
i-1 \\
j-1
\end{array}\right),
\end{aligned}
$$


the binomial coefficients being evaluated over characteristic 2 and $\oplus$ being direct sum of matrices. We take $\mathscr{C}_{0}^{n}(\omega)$ to be the extension with

$$
\lambda(X)=\omega^{2 n+2}\left(M_{1} \oplus M_{2}\right)
$$

and otherwise follow Conlon's choice for the particular extension of $\mathscr{L}$ which will be labelled $\overline{\mathscr{L}}^{0}$.

If $\mathscr{L}$ is an $\mathscr{F} \mathscr{V}$-representation module with stabilizer $\mathscr{P}$ in $\mathscr{N}_{k}$, and $\mathscr{X}$ is as before, the following conditions hold:

$$
\begin{aligned}
& \text { if } X \in \mathscr{S}, \mathscr{V} \unlhd \mathscr{A}_{4} \unlhd \mathscr{A}_{4} \times \mathscr{X} \unlhd \mathscr{S} ; \\
& \text { if } X \notin \mathscr{S}, \quad \mathscr{V} \unlhd \mathscr{V} \times \mathscr{X} \unlhd \mathscr{S} ;
\end{aligned}
$$

in both series equality holds in the last place if and only if $T \notin \mathscr{S}$.

Suppose $\mathscr{F} \mathscr{X} \cong \oplus \mathscr{X}_{i}$ is a decomposition into indecomposable left ideals. Then, from $(7), \mathscr{L}^{\mathscr{r} \times \mathscr{X}} \cong \oplus \mathscr{X}_{i} \otimes_{\mathscr{F}} \mathscr{L}$ is a decomposition into indecomposables. If $X \in \mathscr{S}$ then in every case

so

$$
\mathscr{L}^{\mathscr{A}_{4}} \cong \overline{\mathscr{L}}^{0} \oplus \overline{\mathscr{L}}^{1} \oplus \overline{\mathscr{L}}^{2},
$$

so using (7),

$$
\mathscr{L}^{x_{1} \times x} \cong \bigoplus_{a=0}^{2}\left(\overline{\mathscr{L}}^{a}\right)^{\mathscr{d}_{1} \times x},
$$

$$
\mathscr{L}^{\mathscr{A}_{1} \times \mathscr{X}} \cong \bigoplus_{a=0}^{2} \underset{i}{\oplus} \mathscr{X}_{i} \otimes_{\mathscr{F}_{4}} \overline{\mathscr{L}}^{a}
$$

Again the direct summands are indecomposable.

Let $\mathscr{M}$ be one of the direct summands in this last decomposition. $\mathscr{S} /\left(\mathscr{A}_{4} \times \mathscr{X}\right)$ has order 1 or 2 , and so its group algebra over $\mathscr{F}$ is indecomposable. So $\mathscr{M}^{\mathscr{S}}$ is indecomposable. A similar result holds if $X \notin \mathscr{S}$. We have the following decompositions into indecomposables:

$$
\mathscr{L}^{\mathscr{S}} \cong \begin{cases}\oplus_{i}\left(\mathscr{X}_{i} \otimes_{\mathscr{F}} \mathscr{L}\right) & \text { if } X \notin \mathscr{S} \\ \oplus_{i=0}^{2}\left(\mathscr{X}_{i} \otimes_{\mathscr{F} \mathscr{A}_{4}} \overline{\mathscr{L}}^{a}\right)^{\mathscr{S}} & \text { if } X \in \mathscr{S}\end{cases}
$$

The calculation of the isomorphism-classes of $\mathscr{V}$-projective $\mathscr{F} \mathscr{N}_{k}$-representation modules is now an easy task, using the last part of (6).

For $\mathscr{N}_{7}$ we find that the classes are $A_{0}^{a, b}, A_{n}^{a, b}, B_{n}^{a, b}, C_{n}^{a, b}(\omega), C_{n}^{*, b}(f)$ and $D^{a, b}$, where $a$ and $b$ range through the integers modulo $3, n$ through the positive integers and $f$ through the elements of $\mathscr{F} \cup\{\infty\}$ other than $\omega$ and $\omega^{2}$. The following identities hold: 


$$
\begin{aligned}
& A_{0}^{a, b}=A_{0}^{2 a, 2 b}, \quad A_{n}^{a, b}=A_{n}^{2 a, 2 b}, \quad B_{n}^{a, b}=B_{n}^{2 a, 2 b}, D^{a, b}=D^{2 a, 2 b} \\
& C_{n}^{*, b}(f)=C_{n}^{*, b}(g)=C_{n}^{*, 2 b}(h) \text { when } g=1+\frac{1}{f} \text { or } \frac{1}{1+f}, \\
& h=\frac{1}{f} \text { or } \frac{f}{1+f} \text { or } 1+f^{5}
\end{aligned}
$$

The classes for $\mathcal{N}_{B}$ are just those for $\mathscr{N}_{7}$ with all reference to $b$ dropped (we write, for example, $A_{0}^{a}, C_{n}^{*}(f)$ ) and with $L^{a}=L^{d}$ whenever $L^{a, 0}=L^{d, 0}$. We use the convention that

$$
\left(L^{a, b}\right)_{\mathcal{N}}=L^{a}, \quad\left(L^{a}\right)_{\mathscr{N}_{4}}=L^{a}+L^{2 a} .
$$

The representation matrices for typical members of the $\mathscr{F}_{\mathscr{N}_{7} \text {-classes }}$ are shown in (10) in terms of the corresponding $\mathscr{F} \mathscr{A}_{4}$ representation with superscript 0 . These corresponding representation matrices are denoted by $\lambda$. The table also shows the $\mathscr{F} \mathscr{V}$-class from which each $\mathscr{F}_{\mathcal{N}} \mathscr{N}_{7}$-class is obtained and the dimension of square block-matrices involved. The $\mathscr{F}_{\mathbb{N}_{6}}$ representations are found by deleting the matrix for $Y$.

\begin{tabular}{clll}
\hline $\mathscr{F} \mathscr{N}_{\gamma \text {-class }}$ & $A_{n}^{a, b}, B_{n}^{a, b}$ & $C_{n}^{a, b}(\omega)$ & $C_{n}^{*, b}(f)$ \\
\hline $\begin{array}{l}\text { Corresponding } \\
\text { F } \mathscr{V}_{\text {-class }}\end{array}$ & $A_{n}, B_{n}$ & $C_{n}(\omega)$ & $C_{n}(f)$ \\
\hline $\begin{array}{c}\text { Block size } \\
\text { Matrix for }\end{array}$ & $2 n+1$ & $2 n$ & $6 n$ \\
\hline$U$ & {$\left[\begin{array}{lll}\lambda(U) & 0 \\
0 & \lambda(V)\end{array}\right]$} & {$\left[\begin{array}{lll}\lambda(U) & 0 \\
0 & \lambda(V)\end{array}\right]$} & {$\left[\begin{array}{ll}\lambda(U) & 0 \\
0 & \lambda(V)\end{array}\right]$} \\
\hline$V$ & {$\left[\begin{array}{ll}\lambda(V) & 0 \\
0 & \lambda(U)\end{array}\right]$} & {$\left[\begin{array}{ll}\lambda(V) & 0 \\
0 & \lambda(U)\end{array}\right]$} & {$\left[\begin{array}{ll}\lambda(V) & 0 \\
0 & \lambda(U)\end{array}\right]$} \\
\hline$X$ & {$\left[\begin{array}{lll}\omega^{a} \lambda(X) & 0 \\
0 & & \omega^{2 a} \lambda(X)^{2}\end{array}\right]$} & {$\left[\begin{array}{lll}\omega^{a} \lambda(X) & 0 \\
0 & \omega^{2 a} \lambda(X)^{2}\end{array}\right]$} & {$\left[\begin{array}{ll}\lambda(X) & 0 \\
0 & \lambda(X)^{2}\end{array}\right]$} \\
\hline$T$ & {$\left[\begin{array}{ll}0 & I \\
I & 0\end{array}\right]$} & {$\left[\begin{array}{ll}0 & I \\
I & 0\end{array}\right]$} \\
\hline$Y$ & {$\left[\begin{array}{ll}\omega^{b} I & 0 \\
0 & \omega^{2 b} I\end{array}\right]$} & {$\left[\begin{array}{ll}\omega^{b} I & 0 \\
0 & \omega^{2 b} I\end{array}\right]$} & {$\left[\begin{array}{ll}0 & I \\
I & 0\end{array}\right]$} \\
\hline
\end{tabular}

5 In particular $C_{n}^{*, b}(1)=C_{n}^{*, 2 b}(1)=C_{n}^{*, b}(0)=C_{n}^{*, b}(\infty)$ 


\section{Multiplication of module classes}

The multiplication of module classes is defined in the usual way: If $L$ is $\{\mathscr{L}\}$ and $M$ is $\{\mathscr{M}\}$, then $L M$ is $\{\mathscr{L} \otimes \mathscr{M}\}$.

We shall consider the multiplication of $\mathscr{F} \mathscr{N}_{6}$-classes modulo $W_{\mathscr{G}}(\mathscr{G})$. The effect is that of putting $D^{a}=0$, since $W_{\mathscr{G}}(\mathscr{G})$ is a direct summand of $A_{\mathscr{X}}(\mathscr{G})$ for any $\mathscr{G}$ and any $\mathscr{H} \leqq \mathscr{G},{ }^{6}$ so there is no confusion in writing $L$ for $L+W_{,}\left(\mathscr{N}_{6}\right)$.

The multiplications for $\mathscr{V}$ and $\mathscr{A}_{4}$ are given in propositions 2-4 of $[1]^{7}$ and equations (6), (7), (9) and (11)-(16) of [3]. However we can be more specific about $\bar{C}_{n}^{a}(\omega)$. The convention of Section 4 yields upon a direct calculation the following result when $n \geqq m \geqq 1$ :

$$
\begin{array}{rlrl}
\bar{C}_{m}^{0}(\omega) \bar{C}_{n}^{0}(\omega) & =2 \bar{C}_{m}^{0}(\omega) & \text { if } n \equiv 2(\bmod 3) \\
& =\bar{C}_{m}^{1}(\omega)+\bar{C}_{m}^{2}(\omega) \text { if } n \neq 2(\bmod 3) .
\end{array}
$$

(This replaces equations (13) and (14) of [3].)

From these results, the distributive law and the law

$$
\mathscr{L}^{\mathscr{N}_{0}} \otimes \mathscr{M} \cong\left(\mathscr{L} \otimes \mathscr{M}_{\mathscr{A}_{4}}\right)^{\mathcal{H}_{0}},
$$

where $\mathscr{L}$ is an $\mathscr{F} \mathscr{A}_{4}$-representation module and $\mathscr{M}$ is an $\mathscr{F} \mathscr{N}_{6}$-representation module, we can calculate the products of $\mathscr{F} \mathscr{N}_{6}$-classes. We introduce two points of notation: we write $L^{\mathcal{N}_{0}}$ for the class $\left\{\overline{\mathscr{L}}^{\mathcal{V}_{b}}\right\}$; and we write $f \approx g$ whenever $f \sim g$ or $f \sim 1 / g$, that is whenever $f$ and $g$ are members of the same set of cross-ratios. Example calculations are

$$
\begin{aligned}
A_{n}^{a} A_{m}^{b} & =\left[\left(A_{n}^{a}+A_{n}^{2 a}\right) A_{m}^{b}\right]^{\mathcal{N}_{0}} \\
& =\left(A_{n+m}^{a+b}+\bar{A}_{n+m}^{2 a b}\right)^{\mathcal{N}_{0}} \\
& =A_{n+m}^{a+b}+A_{n+m}^{2 a+b}
\end{aligned}
$$

and, if $m \leqq n$ and $f \not \approx 1$,

except

$$
\begin{aligned}
C_{n}^{*}(f) C_{m}^{*}(g) & =\left[\left(\bar{C}_{n}^{*}(f)+\bar{C}_{n}^{*}(1 / f)\right) \bar{C}_{m}^{*}(g)\right]^{\mathcal{N}_{0}} \\
& = \begin{cases}2 C_{m}^{*}(f) & \text { if } f \approx g, \\
0 & \text { if } f \not g,\end{cases}
\end{aligned}
$$

moreover

$$
C_{\mathbf{1}}^{*}(f) C_{\mathbf{1}}^{*}(f)=C_{\mathbf{2}}^{*}(f) ;
$$

$$
\begin{aligned}
C_{n}^{*}(1) C_{m}^{*}(1) & =\left(2 C_{n}^{*}(1) C_{m}^{*}(1)\right)^{r_{0}} \\
& =4 C_{m}^{*}(1) .
\end{aligned}
$$

Similar calculations yield the following multiplication table:

- This follows from Corollary 5 of [3].

7 There is an error in Bašev's work - see p. 88 of [3]. 


\begin{tabular}{|c|c|c|c|c|}
\hline$m \leqq n$ & $A_{m}^{b}$ & $B_{m}^{b}$ & $C_{m}^{b}(\omega)$ & $C_{m}^{*}(g), g \not \approx \omega$ \\
\hline$A_{n}^{a}$ & $A_{n+m}^{a+b}+A_{n+m}^{2 a+b}$ & $A_{n-m}^{a+b}+A_{n-m}^{2 a+b}$ & $C_{m}^{a+b+n}(\omega)+C_{m}^{2 a+b+n}(\omega)$ & $2 C_{m}^{*}(g)$ \\
\hline$B_{n}^{a}$ & $B_{n-m}^{a+b}+B_{n-m}^{2 a+b}$ & $B_{n+m}^{a+b}+B_{n+m}^{2 a+b}$ & $C_{m}^{a+b+2 n}(\omega)+C_{m}^{2 a+b+2 n}(\omega)$ & $2 C_{m}^{*}(g)$ \\
\hline \multirow[t]{5}{*}{$\overline{C_{n}^{a}(\omega)}$} & $C_{n}^{a+b+m}(\omega)$ & $c_{n}^{a+b+2 m}(\omega)$ & $c_{2}^{a+b}(\omega)$ if $m=n=1$ & \\
\hline & $+C_{n}^{a+2 b+m}(\omega)$ & $+C_{n}^{a+2 b+2 m}(\omega)$ & $2 C_{m}^{a+b}(\omega)$ if $n \equiv 2(\bmod 3)$ & 0 \\
\hline & & & $C_{m}^{a+b+1}(\omega)+C_{m}^{a+b+2}(\omega)$ & \\
\hline & & & if $n \neq 2(\bmod 3)$ & \\
\hline & & & when $n>1$ & \\
\hline \multirow{4}{*}{$\begin{array}{l}C_{n}^{*}(f) \\
f \not \omega \omega\end{array}$} & $2 C_{n}^{*}(f)$ & $2 C_{n}^{*}(f)$ & 0 & 0 if $f \not \approx g$ \\
\hline & & & & $4 C_{m}^{*}(1)$ if $f \approx g \approx 1$ \\
\hline & & & & $2 C_{m}^{*}(f)$ if $n \neq 1$ \\
\hline & & & & $\begin{array}{l}C_{2}^{*}(f) \text { if } m=n=1 \\
\text { when } f \approx g \not \approx 1\end{array}$ \\
\hline
\end{tabular}

We see from (12) that $\frac{1}{2} A_{0}^{0}$ is an identity element for the algebra $\mathscr{R}=A_{\mathscr{Y}}\left(\mathscr{N}_{6}\right) / W_{S}\left(\mathscr{N}_{6}\right)$, and admits of the orthogonal idempotent decomposition $\frac{1}{2} A_{0}^{0}=J_{0}+J_{1}$, where

Then

$$
\begin{aligned}
& J_{0}=\frac{1}{6} A_{0}^{0}+\frac{1}{3} A_{0}^{1}, \\
& J_{1}=\frac{1}{3} A_{0}^{0}-\frac{1}{3} A_{0}^{1}
\end{aligned}
$$

$$
\mathscr{R}=\mathscr{R} J_{0} \oplus \mathscr{R} J_{1} \text {. }
$$

Write $A_{n \alpha}=A_{n}^{0} J_{\alpha}, B_{n \alpha}=B_{n}^{0} J_{\alpha}, C_{n 0}=C_{n}^{0}(\omega) J_{0}, C_{n 1}^{a}=C_{n}^{a}(\omega) J_{1}$. Then the set of these elements (where $\alpha$ is 0 or 1 and $a$ is any integer modulo 3), $J_{0}, J_{1}$ and the distinct $C_{n}^{*}(f)$ together generate $\mathscr{R}$, since

$$
\begin{aligned}
A_{n}^{0} & =A_{n 0}+A_{n 1} \\
B_{n}^{0} & =B_{n 0}+B_{n 1} \\
A_{n}^{1} & =\frac{3}{4} A_{n 0}-\frac{3}{8} A_{n 1} \\
B_{n}^{1} & =\frac{3}{4} B_{n 0}-\frac{3}{8} B_{n 1} \\
C_{n}^{a}(\omega) & =C_{n 0}+C_{n 1}^{a} .
\end{aligned}
$$

Moreover the identity $C_{n 1}^{0}+C_{n 1}^{1}+C_{n 1}^{2}=0$ holds, and so we can $\operatorname{drop} C_{n 1}^{0}$ from the list of generators.

Writing $X_{\alpha}=\frac{1}{2} A_{1 \alpha}$ we see that $A_{n \alpha}=2\left(X_{\alpha}\right)^{n}$ and $B_{n \alpha}=2\left(X_{\alpha}\right)^{-n}$, 
so the set generated by all the $A_{n \alpha}$ and $B_{n \alpha}$ for a given $\alpha$ is isomorphic to $\mathscr{C}\left[X_{\alpha}, 1 / X_{\alpha}\right]$. If we write $\mathscr{A}_{0}$ for the algebra generated by all the $C_{n}^{*}(f)$ and $C_{n 0}$, and $\mathscr{A}_{1}$ for that generated by the $C_{n 1}^{a}$, then

$$
\begin{aligned}
& \mathscr{R} J_{0} \cong \mathscr{C}\left[X_{0}, 1 / X_{0}\right]+\mathscr{A}_{0}, \\
& \mathscr{R} J_{1} \cong \mathscr{C}\left[X_{1}, 1 / X_{1}\right]+\mathscr{A}_{1},
\end{aligned}
$$

where $\mathscr{A}_{\alpha}$ is an ideal of $\mathscr{R} J_{\alpha}$.

We next set up orthogonal idempotents which generate $\mathscr{A}_{0}$ and $\mathscr{A}_{1}$. For $\mathscr{A}_{0}$ we use

$$
\begin{array}{ll}
I_{1}(1)=\frac{1}{4} C_{1}^{*}(1), & \\
I_{n}(1)=\frac{1}{4}\left(C_{n}^{*}(1)-C_{n-1}^{*}(1)\right) & \text { if } n>1, \\
I_{n}(\omega)=\frac{1}{4}\left(C_{20}+(-1)^{n} \sqrt{ } 2 C_{10}\right) & \text { if } n=1 \text { or } 2, \\
I_{n}(\omega)=\frac{1}{2}\left(C_{n 0}-C_{n-1,0}\right) & \text { if } n>2, \\
I_{n}(f)=\frac{1}{4}\left(C_{2}^{*}(f)+(-1)^{n} C_{1}^{*}(f)\right) & \text { if } n=1 \text { or } 2, f \neq 1, \\
I_{n}(f)=\frac{1}{2}\left(C_{n}^{*}(f)-C_{n-1}^{*}(f)\right) & \text { if } n>2, f \neq 1 .
\end{array}
$$

In each case, $\mathscr{R} I_{n}(f) \cong \mathscr{C} I_{n}(f)$.

To consider $\mathscr{A}_{1}$ we set

$$
\begin{array}{ll}
K_{n}^{a}=\frac{1}{4}\left(C_{21}^{a}+(-1)^{n} \sqrt{ } 2 C_{11}^{a}\right) & \text { if } n=1 \text { or } 2, \\
K_{n}^{a}=h_{n} C_{n 1}^{a}-h_{n-1} C_{n-1,1}^{a} & \text { if } n>2,
\end{array}
$$

where $h_{n}=\frac{1}{2}$ if $n \equiv 2(\bmod 3)$ and $h_{n}=-1$ otherwise.

The $K_{n}^{0}$ are orthogonal and $\mathscr{R} K_{n}^{0} \cong \mathscr{C}\left[K_{n}^{0}, K_{n}^{1}, K_{n}^{2}\right]$. Since $K_{n}^{a} K_{n}^{b}=K_{n}^{a+b}$ and $K_{n}^{\mathbf{0}}+K_{n}^{\mathbf{1}}+K_{n}^{\mathbf{2}}=0$ we see that if we put

$$
\begin{aligned}
& L_{n 0}=\frac{1}{3}\left(K_{n}^{0}+u K_{n}^{1}+u^{2} K_{n}^{2}\right), \\
& L_{n 1}=\frac{1}{3}\left(K_{n}^{0}+u^{2} K_{n}^{1}+u K_{n}^{2}\right),
\end{aligned}
$$

where $u$ is a primitive cube root of unity in $\mathscr{C}$, then

$$
\mathscr{R} K_{n}^{\mathbf{0}}=\mathscr{R} L_{n \mathbf{0}} \oplus \mathscr{R} L_{n \mathbf{1}} \text { and } \mathscr{R} L_{n \alpha} \cong \mathscr{C} L_{n \alpha} \text {. }
$$

From these we can find the structure of $\mathscr{R} . A_{\mathfrak{r}}\left(\mathscr{N}_{6}\right) \cong \mathscr{R} \oplus W_{\mathfrak{s}}\left(\mathscr{N}_{6}\right)$, and $W_{f}\left(\mathscr{N}_{6}\right) \cong \mathscr{C} \oplus \mathscr{C}$, so $A_{\mathfrak{r}}\left(\mathscr{N}_{6}\right)$ has the following form to within isomorphism:

$$
\begin{aligned}
A_{\mathscr{r}}\left(\mathscr{N}_{6}\right) \cong & \left\{\mathscr{C}\left[X_{0}, 1 / X_{0}\right]+\oplus_{1} \mathscr{C} I_{n}(f)\right\} \\
& \oplus\left\{\mathscr{C}\left[X_{1}, 1 / X_{1}\right]+\oplus_{2} \mathscr{C} L_{n \alpha}\right\} \\
& \oplus\{\mathscr{C} \oplus \mathscr{C}\}
\end{aligned}
$$

where $\oplus_{1}$ ranges over all positive integers $n$ and all $f \in \mathscr{F} \cup\{\infty\}$ modulo the relation $\approx$, and $\oplus_{2}$ ranges over the positive integers $n$ and $\alpha=0,1$. The $I_{n}(f)$ and the $L_{n \alpha}$ are sets of orthogonal idempotents, and 


$$
\begin{aligned}
X_{0} I_{n}(f) & =I_{n}(f), \\
X_{1} L_{n \alpha} & =u^{2 \alpha+2} L_{n \alpha} .
\end{aligned}
$$

We work similarly in the $\mathscr{N}_{7}$ case. The $\mathscr{V}$-projective $\mathscr{F} \mathscr{N}_{7}$-representation module classes are considered modulo $W_{s}\left(\mathscr{N}_{7}\right)$, and find the following (14)

\begin{tabular}{|c|c|c|c|c|}
\hline$m \leqq n$ & $A_{m}^{b, d}$ & $B_{m}^{b, d}$ & $C_{m}^{b, d}(\omega)$ & $C_{m}^{*, d}(g), g \not \approx \omega$ \\
\hline \multirow[t]{2}{*}{$A_{n}^{a, c}$} & & & & $C_{m}^{*, c+d}(g)+C_{m}^{*, 2 c+d}(g)$ \\
\hline & $+A_{m+n}^{a+2 b, c+2 d}$ & $+A_{n-m}^{a+2 b, c+2 d}$ & $+C_{m}^{n+2 a+b, 2 c+d}(\omega)$ & \\
\hline \multirow[t]{2}{*}{$B_{n}^{a, c}$} & $B_{n-m}^{a+b, c+d}$ & $B_{n+m}^{a+b, c+d}$ & $C_{m}^{2 n+a+b, c+d}(\omega)$ & $C_{m}^{*, c+d}(g)+C_{m}^{*, 2 c+d}(g)$ \\
\hline & $+B_{n-m}^{a+2 b, c+2 d}$ & $+B_{n+m}^{a+2 b, c+2 d}$ & $+C_{m}^{2 n+2 a+b, 2 c+d}(\omega)$ & \\
\hline \multirow[t]{4}{*}{$C_{n}^{a, c}(\omega)$} & $\begin{array}{l}C_{n}^{m+a+b, c+d}(\omega) \\
\quad+C_{n}^{m+a+2 b, c+2 d}(\omega)\end{array}$ & $\begin{array}{l}C_{n}^{2 m+a+b, c+d}(\omega) \\
\quad+C_{n}^{2 m+a+2 b, c+2 d}(\omega)\end{array}$ & if $\begin{array}{r}n=m=1 \\
C_{2}^{a+b, c+d}(\omega)\end{array}$ & 0 \\
\hline & & & $\begin{array}{l}\text { if } n \neq 1, n \equiv 2(\bmod 3) \\
2 C_{m}^{a+b, c+d}(\omega)\end{array}$ & \\
\hline & & & if $\begin{array}{c}n \neq 1, n \neq 2(\bmod 3) \\
C_{m}^{a+b+1, c+d}(\omega)\end{array}$ & \\
\hline & & & $+C_{m}^{a+b+2, c+a}(\omega)$ & \\
\hline
\end{tabular}
multiplication table for $\mathscr{S}=A_{\mathscr{V}}\left(\mathscr{N}_{7}\right) / W_{\mathcal{F}}\left(\mathscr{N}_{7}\right)$ :

$C_{n}^{*, c}(f) \quad C_{n}^{*, c+d}(f)+C_{n}^{*, c+2 d}(f) \quad C_{n}^{*, c+d}(f)+C_{n}^{*, c+2 d}(f) \quad 0$

0 if $f \not \approx g$

$t \not \approx \omega$

\begin{tabular}{l}
$2\left(C_{m}^{*, c+d}(1)+C_{m}^{*, 2 c+d}(1)\right)$ \\
if $f \approx g \approx 1$ \\
\hline$C_{2}^{c+d}(f)$ if $f \approx g \not 1$, \\
$m=n=1$ \\
\hline $2 C_{m}^{c+d}(f)$ if $f \approx g \not \approx 1$, \\
$n \neq 1$
\end{tabular}

Write $S=A_{0}^{0,1}+A_{0}^{1,0}+A_{0}^{1,2}$. The identity element $\frac{1}{2} A_{0}^{0,0}$ of $\mathscr{S}$ admits of an orthogonal idempotent decomposition:

$$
\begin{aligned}
\frac{1}{2} A_{0}^{0,0} & =J_{0}+J_{10}+J_{01}+J_{11}+J_{12}, \\
J_{0} & =\frac{1}{18}\left(A_{0}^{0,0}+2 S\right) \\
J_{a b} & =\frac{1}{9}\left(A_{0}^{0,0}+3 A_{0}^{a, b}-S\right)
\end{aligned}
$$

We proceed much as before. It is clear that $\mathscr{S}$ is generated by the $J_{x}$ and the elements 


$$
\begin{array}{rlrl}
A_{n x} & =A_{n}^{0,0} J_{x} & B_{n x} & =A_{n}^{0,0} J_{x} \\
C_{n, 0}(f) & =C_{n}^{*, 0}(f) J_{0} & C_{n, 10}^{a}(f) & =C_{n}^{*, a}(f) J_{10} \\
C_{n, 0}(\omega) & =C_{n}^{0,0}(\omega) J_{0} & C_{n, 10}^{a}(\omega) & =C_{n}^{0, a}(\omega) J_{10} \\
C_{n, x}^{a}(\omega) & =C_{n}^{a, 0}(\omega) J_{x}(x \neq 0,10), &
\end{array}
$$

where $a$ ranges through the integers modulo $3, x$ through $\{0,01,10,11,12\}$ and $f$ through the non-equivalent members of $\mathscr{F}$ other than $\omega$ under the relation $\approx$. and

Putting $Y_{x}=\frac{1}{2} A_{1 x}$ we obtain as before $A_{n, x}=2\left(Y_{x}\right)^{n}, B_{n, x}=2\left(Y_{x}\right)^{-n}$,

$$
\mathscr{S} J_{x} \cong \mathscr{C}\left[Y_{x}, 1 / Y_{x}\right]+\mathscr{B}_{x},
$$

where $\mathscr{B}_{x}$ is an ideal. We then write

$$
\begin{array}{rlrl}
I_{n}(f) & =\frac{1}{4}\left(C_{2,0}(f)+(-1)^{n} \sqrt{ } 2 C_{1,0}(f)\right) & & \text { if } n=1 \text { or } 2, \\
I_{n}(f) & =\frac{1}{2}\left(C_{n, 0}(f)-C_{n-1,0}(f)\right) & & \text { if } n>2, \\
K_{n, 10}^{a}(f) & =\frac{1}{4}\left(C_{2,10}^{a}(f)+(-1)^{n} \sqrt{ } 2 C_{1,10}(f)\right) & \text { if } n=1 \text { or } 2 \text { and } f \neq 1, \\
K_{n, 10}^{a}(f) & =\frac{1}{2}\left(C_{n, 10}^{a}(f)-C_{n-1,10}^{a}(f)\right) & & \text { if } n>2 \text { and } f \neq 1, \\
K_{1,10}^{0}(1) & =\frac{1}{4} C_{1,10}^{0}(1) & \\
K_{n, 10}^{0}(1) & =\frac{1}{4}\left(C_{n, 10}(1)-C_{n-1,10}(1)\right) & \text { if } n \neq 1, \\
K_{n, x}^{a}(\omega) & =\frac{1}{4}\left(C_{2, x}^{a}(\omega)+(-1)^{n} \sqrt{ } 2 C_{1, x}^{a}(\omega)\right) & \text { if } n=1 \text { or } 2 \\
K_{n, x}^{a}(\omega) & =h_{n} C_{n, x}^{a}(\omega)-h_{n-1} C_{n-1, x}^{a}(\omega) & \text { if } n>2,
\end{array}
$$

where $x=01,11$ or 12 and $h_{n}$ is defined as before. It is found that $\left\{I_{n}(f)\right\}$, $\left\{K_{n, 10}^{\mathbf{0}}(f)\right\}$ and $\left\{K_{n, x}^{\mathbf{0}}(\omega)\right\}$ are sets of orthogonal idempotents generating $\mathscr{B}_{0}, \mathscr{B}_{10}$ and $\mathscr{B}_{x} \cdot \mathscr{S} I_{n}(f) \cong \mathscr{C} I_{n}(f)$ and $\mathscr{S} K_{n, 10}^{0}(1) \cong \mathscr{C} K_{n, 10}^{0}(1) ;$ in the other cases we find

so we put

$$
\mathscr{S} K_{n, x}^{\mathbf{0}}(f) \cong \mathscr{C}\left[K_{n, x}^{\mathbf{0}}(f), K_{n, x}^{\mathbf{1}}(f), K_{n, x}^{\mathbf{2}}(f)\right]
$$

$$
L_{n, \alpha, x}(f)=K_{n, x}^{0}(f)+u^{\alpha+1} K_{n, x}^{1}(f)+u^{2 \alpha+2} K_{n, x}^{2}(f) .
$$

For convenience write $L_{n, \mathbf{0 , 1 0}}(1)=K_{n, 10}^{\mathbf{0}}(1)$.

Proceeding as before we find $A_{\mathscr{r}}\left(\mathscr{N}_{7}\right)$ is

$$
\begin{aligned}
A_{r}\left(\mathscr{N}_{7}\right) & \cong\left\{\mathscr{C}\left[Y_{0}, 1 / Y_{0}\right]+\oplus_{1} \mathscr{C} I_{n}(f)\right\} \\
& \oplus\left\{\mathscr{C}\left[Y_{10}, 1 / Y_{10}\right]+\oplus_{2} \mathscr{C} L_{n, \alpha, 10}(f)\right\} \\
& \oplus \oplus_{3}\left\{\mathscr{C}\left[Y_{x}, 1 / Y_{x}\right]+\oplus_{4} L_{n, x, x}(\omega)\right\} \\
& \oplus\{\mathscr{C} \oplus \mathscr{C} \oplus \mathscr{C} \oplus \mathscr{C} \oplus \mathscr{C}\}
\end{aligned}
$$

where $\oplus_{1}$ is over all elements $f$ of $\mathscr{F}, \operatorname{modulo} \approx$, and all positive integers $n$,

$\oplus_{2}$ is as $\oplus_{1}$, and also over $\alpha=0,1$, except for the case $f=1, \alpha=1$,

$\oplus_{3}$ is over $x=01,10,12$,

$\oplus_{4}$ is over all $n \geqq 1$ and $\alpha=0,1$, and we have 


$$
\begin{aligned}
Y_{0} I_{n}(f) & =I_{n}(f) \\
Y_{10} L_{n, \alpha, 10}(f) & =L_{n, \alpha, 10}(f) \\
Y_{x} L_{n, \alpha, x}(\omega) & =u^{2 \alpha+2} L_{n, \alpha, x}(\omega) .
\end{aligned}
$$

The classes of $\mathscr{Z}_{2}$-projective $\mathscr{V}$-modules are $D$ and $C_{1}(\mathbf{1})$. Therefore

$$
\begin{aligned}
& A_{\mathscr{T}_{2}}\left(\mathscr{N}_{6}\right) \cong \mathscr{C} I_{1}(1) \oplus \mathscr{C} \oplus \mathscr{C} \\
& A_{\mathscr{T}_{2}}\left(\mathscr{N}_{7}\right) \cong \mathscr{C} I_{1,0}(1) \oplus \mathscr{C} L_{1,0,10}(1) \oplus \mathscr{C} \oplus \mathscr{C} \oplus \mathscr{C} \oplus \mathscr{C} \oplus \mathscr{C},
\end{aligned}
$$

Thus $W_{\curlyvee}\left(\mathscr{N}_{6}\right) \cong \mathscr{R} / \mathscr{C} I_{1}(1) . I_{1}(1) \in \mathscr{R} J_{0}$, so we need only consider this factor. It can be split into two components, one of which is $\mathscr{C} I_{1}(1)$, by the idempotent decomposition

$$
\begin{aligned}
J_{0} & =I_{1}(1)+\left(J_{0}-I_{1}(1)\right) \\
& =I_{1}(1)+J_{0}, \text { say. }
\end{aligned}
$$

If we write $\bar{X}_{0}=X_{0} J_{0}$, then the decomposition of $\mathscr{R} J_{0}$ is just that of $\mathscr{R} J_{0}$ with $X_{0}$ replaced by $\bar{X}_{0}$, and with the case $f=1, n=1$, dropped from the summation. Notice that $\bar{X}_{0} I_{n}(f)=X_{0} I_{n}(f)$ except when $n=1$ and $f=1$. The same considerations apply to the $\mathscr{N}_{7}$ case. Therefore when $k=6$ or 7 the form of $W_{v}\left(\mathscr{N}_{k}\right)$ is just that given in (13) or (15), provided that the final term consisting of copies of $\mathscr{C}$ is omitted and that the case $n=1, f=1$, is dropped from all direct sums where it occurs.

\section{Semisimplicity}

It is now easy to see that $W_{\mathscr{r}}\left(\mathscr{N}_{6}\right)$ and $W_{\mathscr{r}}\left(\mathscr{N}_{7}\right)$ are semisimple. If $\mathscr{A}_{1}$ is any algebra of the form

$$
\mathscr{A}_{1}=\mathscr{C}[X, 1 / X]+\mathscr{B}
$$

where $\mathscr{B}$ is an ideal of the form $\oplus \mathscr{C} I_{r}$ with $r$ ranging through some indexing set, then $\mathscr{A}_{1}$ is semisimple. ${ }^{8}$ It is clear that if $\mathscr{A}_{1} \oplus \mathscr{A}_{2} \oplus \cdots$ is a finite sum of semisimple algebras then it is semisimple. But both $W_{\mathscr{r}}\left(\mathscr{N}_{6}\right)$ and $W_{\downarrow}\left(\mathscr{N}_{7}\right)$ are of this form, where each $\mathscr{A}_{i}$ has the form of $\mathscr{A}_{1}$ in (16). Therefore we have the following result.

(17) Theorem. $W_{\mathscr{V}}\left(\mathscr{N}_{6}\right)$ and $W_{\boldsymbol{V}}\left(\mathscr{N}_{7}\right)$ are semisimple.

\section{References}

[1] V. A. Bašev, 'Representations of the group $Z_{\mathbf{2}} \times Z_{2}$ into a field of characteristic 2', (Russian) Dokl. Akad. Nauk SSSR 141 (1961), 1015-1018.

[2] S. B. Conlon, 'Twisted group algebras and their representations', J. Aust. Math. Soc. 4 (1964), 152-173.

8 The proof is an easy generalization of the proof of the Theorem on p. 90 of [3]. 
[3] S. B. Conlon, 'Certain representation algebras', J. Aust. Math. Soc. 5 (1965), $83-99$.

[4] S. B. Conlon, 'The modular representation algebra of groups with Sylow 2-subgroup $Z_{2} \times Z_{2}^{\prime}, J$. Aust. Math. Soc. 6 (1966), 76-88.

[5] S. B. Conlon, 'Structure in representation algebras', J. of Algebra 5 (1967), $274-279$.

[6] J. A. Green, 'A transfer theorem for modular representations', J. of Algebra 1 (1964), 7384.

[7] A. Heller and I. Reiner, 'Indecomposable representations', Illinois J. Math. 5 (1961), 314323.

[8] M. F. O'Reilly, 'On the semisimplicity of the modular representation algebra of a finite group', Illinois J. Math. 9 (1965), 261-276.

[9] H. Wielandt, Finite permutation groups (Academic Press, 1964).

\section{La Trobe University}

Melbourne 